\title{
REFLEXÕES SOBRE O ENFRENTAMENTO DO CORONAVÍRUS DISEASE 2019: DIÁLOGOS VIRTUAIS COM GESTANTES
}

\section{REFLECTIONS ON COPING WITH CORONAVIRUS DISEASE 2019: VIRTUAL DIALOGUES WITH PREGNANT WOMEN}

\section{REFLEXIONES SOBRE EL ENFRENTAMIENTO DE LA ENFERMEDAD DE CORONAVIRUS 2019: DIÁLOGOS VIRTUALES CON MUJERES EMBARAZADAS}

Jeane Barros de Souzaㄹ, Ivonete Terezinha Schülter Buss Heidemann², Carine Vendruscolo³, Erica de Brito Pitilin4, Eleine Maestri5 Valéria Silvana Faganello Madureira ${ }^{6}$

\section{RESUMO}

Objetivo: Relatar a vivência de um Círculo de Cultura virtual, com reflexões sobre o enfrentamento da CoVid-19 e as repercussões para a saúde das gestantes. Método: Relato de experiência de um Círculo de Cultura virtual utilizando o referencial teóricometodológico de Paulo Freire. Participaram dez gestantes de diferentes localidades do Brasil. Os diálogos foram conduzidos com ludicidade, estabelecendo analogia com o desenvolvimento da gravidez: o embrião foi considerado Investigação Temática; o feto, Codificação e Descodificação; o nascimento, Desvelamento Crítico. Resultados: Os diálogos e reflexões emergentes possibilitaram que as gestantes aliviassem suas angústias e medos e ressignificassem suas atitudes, motivando-se mutuamente em prol da saúde, diante das adversidades geradas pela pandemia. Conclusão: O Círculo de Cultura virtual proporcionou interação às gestantes, mesmo estando fisicamente afastadas, configurando-se como possibilidade inovadora na assistência individual e coletiva, nos territórios de atuação das equipes que operam na atenção à saúde, para promover o cuidado no enfrentamento da doença.

Descritores: Gravidez; Quarentena; Promoção da saúde; Saúde da Mulher.

\begin{abstract}
Objective: To report the experience of a virtual Culture Circle, with reflections on coping with CoVid-19 and the repercussions for the health of pregnant women. Method: Experience report of a virtual Culture Circle using Paulo Freire's theoretical-methodological framework. Ten pregnant women from different locations in Brazil participated in this study. The dialogues were conducted with playfulness, establishing an analogy with the development of pregnancy: the embryo was considered thematic investigation; the fetus, Encoding and Decoding; the birth, Critical Unveiling. Results: The dialogues and emerging reflections made it possible for pregnant women to relieve their anxieties and fears and redefine their attitudes, motivating each other in favor of health, in the face of the adversities caused by the pandemic. Conclusion: The Virtual Culture Circle provided interaction for pregnant women. Despite their distance, this experience configures as an innovative possibility in individual and collective assistance for teams that operate in health care to promote the care in coping with the disease.
\end{abstract}

Descriptors: Pregnancy; Quarantine; Health Promotion; Women's Health.

\section{RESUMEN}

Objetivo: Informar sobre la experiencia de un Círculo de Cultura virtual, con reflexiones sobre cómo enfrentar CoVid-19 y las repercusiones para la salud de las mujeres embarazadas. Método: Informe de experiencia de un Círculo de Cultura virtual utilizando el marco teórico-metodológico de Paulo Freire. Participaron diez mujeres embarazadas de diferentes lugares de Brasil. Los diálogos se llevaron a cabo de una manera lúdica, estableciendo una analogía con el desarrollo del embarazo: el embrión se consideraba una investigación temática; el feto, codificación y decodificación; nacimiento, desvelación crítica. Resultados: Los diálogos y las reflexiones emergentes permitieron a las mujeres embarazadas aliviar sus ansiedades y temores, y redefinir sus actitudes, motivarse mutuamente a favor de la salud, frente a las adversidades generadas por la pandemia. Conclusión: El Círculo de Cultura Virtual proporcionó interacción para las mujeres embarazadas, a pesar de que estaban físicamente separadas, configurándose como una posibilidad innovadora en la asistencia individual y colectiva, en los territorios de los equipos que operan en la atención médica, para promoverla en el enfrentamiento a la enfermedad.

Descriptores: Embarazo; Cuarentena; Promoción de la Salud; Salud de la Mujer.

${ }^{1}$ Doutora em Ciências. Docente do curso de Enfermagem da Universidade Federal da Fronteira Sul. 2PhD em enfermagem Saúde Pública. Professor associado do Departamento e do Programa de Pós Graduação em Enfermagem da Universidade Federal de Santa Catarina. ${ }^{3}$ Doutora em Enfermagem. Professora titular da PósGraduação em Enfermagem da Universidade do Estado de Santa Catarina. ${ }^{4}$ Doutoranda do Programa de Pós-Graduação em Enfermagem da Universidade Federal de São Paulo. Docente do Curso de Graduação em Enfermagem na Universidade Federal da Fronteira Sul, Campus Chapecó. ${ }^{5}$ Doutora em Enfermagem. Docente do Curso de Graduação em Enfermagem da Universidade Federal Fronteira Sul, Campus Chapecó. ${ }^{6}$ Doutora em Enfermagem, Docente do curso de Enfermagem da Universidade Federal da Fronteira Sul. 


\section{INTRODUÇÃO}

A gestação é um fenômeno fisiológico e deve ser compreendida e vivenciada por mulheres grávidas e pelas equipes de saúde como parte de uma experiência de vida saudável, envolta em grandes expectativas e com mudanças na vida da mulher, nos mais diferentes contextos. No entanto é uma situação limítrofe, em que a préexistência de qualquer condição clínica de risco materno e/ou fetal aumenta a probabilidade de uma evolução desfavorável, caracterizando essas mulheres como gestantes de alto risco(1).

Para a Organização Mundial da Saúde (OMS), as gestantes merecem cuidados especiais, além de uma assistência pré-natal distinta e uma avaliação fetal criteriosa, já que apresentam maior possibilidade de hospitalização e complicações maternas e fetais ${ }^{(2)}$. Todavia, a partir da imposição de mudanças pela pandemia causada pela Coronavirus Disease 2019 (CoVid-19), esse cuidado tem sido restrito para a maioria das gestantes.

A CoVid-19 emergiu como um grave problema de saúde pública global, que se espalhou rapidamente, a partir da China para outras partes do mundo ${ }^{(3)}$. As evidências apontam que a transmissão de pessoa para pessoa vem se acumulando, sem tratamentos ou vacinas eficazes até o momento e, ainda, com desconhecimento razoável sobre caraterísticas da doença e suas repercussões ${ }^{(4)}$.

Com a CoVid-19 parece aumentar o risco de complicações, no ciclo gravídico puerperal, o manejo dessas mulheres deve ser rigoroso ${ }^{(5)}$. Assim, impôs-se a necessidade de reorganização dos serviços para todas as gestantes, o que resultou no cancelamento e/ou adiamento das consultas de pré-natal, alternando entre consultas on-line, realização de exames laboratoriais e/ou de imagem essenciais e indispensáveis, além do rearranjo no cenário assistencial durante o parto ${ }^{(5)}$.

O período gestacional se tornou crítico, em condições de pandemia, o que se refletiu, por exemplo, nos comportamentos desejados para o período gravídico como a restrição e/ou ausência na participação em grupos de apoio, rodas de conversa, grupos de gestantes, chás de bebê, ensaios fotográficos, entre outros. Lidar com o desconhecido e com as modificações fisiológicas e esperadas, resultantes da gravidez em um contexto de pandemia, em que tudo é novo, é ter uma preocupação redobrada, já que se trata da saúde da mãe e do bebê(6).
Tais modificações psicológicas e emocionais expõem o período gestacional a uma nova demanda e a novos modelos de comportamento, na busca de equilíbrio diante do cenário de restrição social. Embora as gestantes compreendam a necessidade dessas medidas, pode não implicar na diminuição do impacto na saúde e nos sentimentos das mulheres relacionados ao gestar e ao parir em tempos de pandemia.

Considerando que a gravidez é um período complexo e rico de sentidos, os quais só podem ser interpretados dentro do contexto da história particular de cada gestante, é necessário compreender a vivência das mulheres grávidas, no enfrentamento da CoVid-19 e buscar assisti-las neste período. Nesse cenário, em que as possibilidades virtuais de estabelecer relacionamentos sociais ganharam vulto, vislumbrou-se a possibilidade de ampliar a utilização de tecnologias para aproximar as gestantes de maneira virtual e estimular a reflexão sobre a CoVid-19, proporcionando espaços para a promoção da saúde.

Assim, pesquisadoras com experiência na realização do Círculo de Cultura de Paulo Freire, decidiram adaptar a metodologia para um modo virtual, a fim de possibilitar reflexões e promover a saúde de gestantes no enfrentamento da CoVid19. A partir da experiência, emergiu este artigo, com o objetivo de relatar a vivência de um Círculo de Cultura Virtual (CCV), com reflexões sobre o enfrentamento da doença e suas repercussões para a saúde das gestantes.

\section{MÉTODO}

\section{Tipo de Estudo}

Trata-se de um relato de experiência, oriundo de uma ação mediada pelo referencial teórico metodológico de Paulo Freire ${ }^{(7)}$. A experiência foi desenvolvida no âmbito de um projeto de pesquisa aprovado pelo Comitê de Ética em Pesquisa com parecer número 4.068.387. No Itinerário Freireano, há participação de todos os envolvidos nas situações abordadas em diálogo e na busca de possíveis encaminhamentos e decisões. A atividade demanda mediador e participantes como pessoas atuantes que buscam o entendimento da realidade vivenciada ${ }^{(7)}$. O Itinerário de Paulo Freire é adaptado e utilizado para atender aos objetivos pretendidos, mediante três momentos interligados: (1) Investigação Temática, por meio de círculos de investigação; (2) Codificação e Descodificação dos Temas 
Geradores; e (3) Desvelamento Crítico da realidade encontrada, por meio de diálogos críticos com mediação de pesquisadores. Esses momentos ocorreram em ambientes designados como Círculo de Cultura, o qual, neste trabalho, foi realizado virtualmente ${ }^{(8)}$.

O Círculo de Cultura caracteriza-se por ser um grupo de pessoas com o objetivo de discutir temas em comum, em relações horizontalizadas e participativas. Cada pessoa traz suas vivências e experiências e as compartilha, de maneira a protagonizar o diálogo, mediado por um facilitador que problematiza os temas que emergem, no intuito de instigar o conhecimento coletivo $^{(9)}$.

\section{Cenário}

Na vivência da quarentena imposta pela CoVid-19, tornou-se necessário realizar o Círculo de Cultura de forma virtual. Nesse sentido, utilizou-se um aplicativo de celular gratuito, e a interação das participantes foi possível pela câmera do dispositivo eletrônico, mesmo estando elas geograficamente distantes.

Para a concretização do Círculo de Cultura e manutenção de uma relação mais próxima entre as participantes, optou-se por convidar, primeiramente, duas gestantes do convívio social das autoras, a fim de facilitar a interação e o aprofundamento das discussões. Essas gestantes convidaram outras amigas grávidas para integrar o CCV. A atividade foi, então, desenvolvida com dez gestantes.

\section{Coleta de Dados}

O CCV foi realizado, em 12 de abril de 2020, período em que vigorava a quarentena no Brasil, com duração de aproximadamente duas horas e meia. Para o desenvolvimento efetivo do CCV, as gestantes foram orientadas sobre o funcionamento do aplicativo, dois dias antes de sua realização e um teste foi executado, com vista a verificar a conexão, imagem e som com todas as envolvidas no processo. Nesse momento, também, foi solicitado que cada gestante providenciasse um pincel atômico e duas folhas de papel A4 para serem utilizados durante a vivência do CCV. Dia e horário foram agendados de forma que todas pudessem participar.

No dia agendado, foi iniciado o CCV sob mediação de uma enfermeira, doutora, com experiência na condução de Círculo de Cultura. Todas as participantes se apresentaram, expondo seu nome e as semanas de gestação em que se encontravam. Em seguida, a proposta foi compartilhada com as gestantes, detalhando a metodologia de trabalho.

A fim de percorrer as etapas do Itinerário Freireano de maneira concreta para as gestantes, optou-se por realizar uma analogia com o desenvolvimento de uma gravidez que, para ser saudável, depende de sucesso em todas as suas fases até o nascimento da criança. Vale destacar que as três fases do Itinerário Freireano estão interligadas entre si, assim como o desenvolvimento de uma gravidez. A proposta de fazer a travessia do Itinerário, por meio dessa analogia, teve também o propósito de tornar as discussões sobre o enfrentamento da CoVid-19 mais criativas, lúdicas, interativas, dialógicas e convergentes à realidade das gestantes, o que oportunizou a realização de todas as fases do Itinerário, em um único encontro, conforme a Figura 1.

Figura 1 - Itinerário Freireano: analogia com o desenvolvimento da gravidez

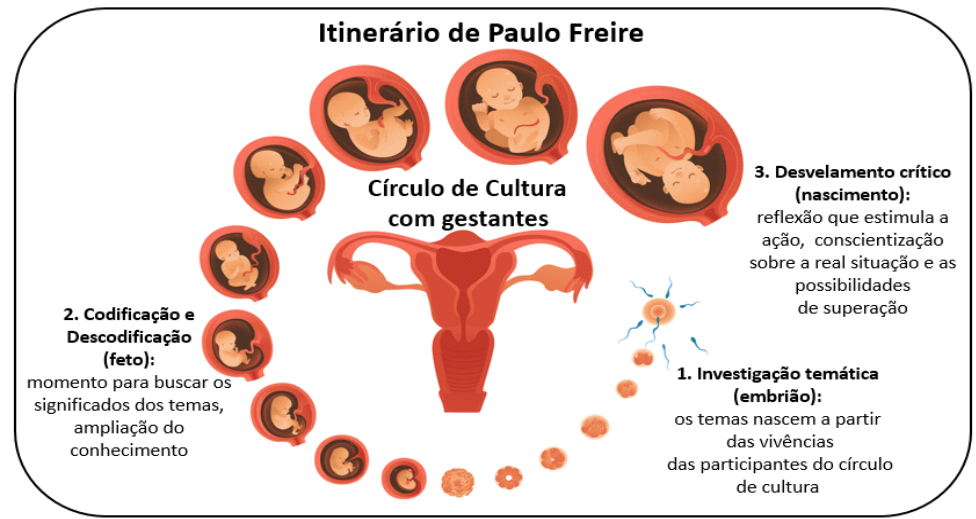

Fonte: Adaptado pelas autoras a partir de https://www.vectorstock.com/royalty-free-vector/human-embryo-development-circleflat-icon-vector-23329044(10) 
A Investigação Temática foi comparada ao embrião, estrutura originária da fertilização de um óvulo por um espermatozoide, o qual se fixa na cavidade uterina da mulher. A mediadora mostrou a imagem de um embrião no útero e apontou que aquele seria o momento para as gestantes se fixarem na temática do enfrentamento da CoVid19 , buscando seus profundos sentimentos diante das suas repercussões no Brasil e no mundo. Para incentivar o diálogo, a mediadora lançou o seguinte questionamento: quais seus sentimentos em relação à CoVid-19? As gestantes escreveram em uma folha A4 seus sentimentos, e todas tiveram a oportunidade de ouvir umas às outras e de serem ouvidas, por meio do diálogo e promovendo a reflexão sobre o mundo concretamente vivido nas ações cotidianas de cada participante, abrindo possibilidades para a sua transformação.

Na fase da Codificação e da Descodificação, que se refere à situação experienciada pelos participantes, a mediadora mostrou a imagem de um feto e abordou que, a partir daquele desenvolvimento, suas características seriam mais evidenciadas e que seus movimentos seriam mais intensos no ventre da mãe. A mediadora convidou as participantes a admirar e refletir sobre os Temas Geradores, percebendo suas características, sua origem e consequências para suas vidas. Assim, as participantes foram instigadas a desvelar uma consciência crítica sobre a gestação saudável diante da CoVid-19. Nesse momento, todas as gestantes exploraram seus medos e transformações, além de outros sentimentos despertados pela necessidade de vivenciar a quarentena no período gestacional e as demais repercussões, com a chegada da pandemia, como questões econômicas, políticas e sociais.

É a partir da situação existencial, do pensar o cotidiano, que se enfrenta a realidade. Assim, na fase do Desvelamento Crítico, ocorreu a conscientização sobre os Temas Geradores investigados, por meio do diálogo e tornando mais concreto um movimento de transformação da realidade vivida. Para tanto, a mediadora apresentou a imagem do útero, no momento do nascimento do bebê, retratando o término da viagem no ventre materno, em que as famílias recebem a criança e, geralmente, atribuem-lhe um nome com um significado especial.

Assim sendo, a mediadora evidenciou que estavam quase chegando ao término do CCV e as convidou a desvelar os aprendizados significativos acerca das vivências no enfrentamento da CoVid19 e/ou sobre a experiência de participar do Círculo de Cultura, incentivando a interação de todas para escrever tais significados em uma folha A4. Enquanto as gestantes escreviam, a mediadora cantou a música 'Tente outra vez', composição de Raul Seixas, acompanhada de violão, a fim de estimular sentimentos e oportunizar a criatividade.

\section{Análise e tratamento dos dados}

O desvelamento dos temas investigados foi realizado, durante todo o processo e com todas as gestantes participantes do Círculo de Cultura, conforme prevê o método de Paulo Freire. Os temas foram ressignificados, por meio das discussões e reflexões, que emergiram no encontro. Essa etapa é um processo contínuo de ação-reflexão-ação, que auxilia na compreensão da realidade ${ }^{(7,9)}$, a partir das discussões que emergem no CCV, no qual as gestantes se empoderaram para promover o enfrentamento da CoVid-19.

\section{RESULTADOS E DISCUSSÃO}

As gestantes tinham idade entre 21 e 36 anos, mencionaram ser de classe média e viviam em diferentes locais do Brasil: quatro eram de Santa Catarina, duas do Rio Grande do Sul, uma de São Paulo, uma do Rio de Janeiro, uma do Paraná e uma do Ceará. As participantes estavam entre a vigésima e trigésima terceira semana de gestação e todas encontravam-se em quarentena.

Após discussão sobre a vivência da quarentena e dos desdobramentos no enfrentamento da CoVid-19, durante a gestação, as participantes da atividade definiram dois Temas Geradores, na fase da Investigação Temática: 1) Preocupações geradas; 2) Planos modificados.

A investigação dos temas constituiu-se em um momento de descobertas daquilo que afetava - dia a dia das participantes, durante a quarentena, adentrando seu universo vocabular e possibilitando a percepção crítica da realidade vívida. Para 'gestar as reflexões' e percorrer a etapa da Codificação e Descodificação, que envolve a apreensão dos temas investigados e o despertar crítico sobre a realidade, as gestantes foram em busca dos significados dessas temáticas. A Descodificação trata de uma análise crítica sobre uma situação que foi codificada, que provoca uma viagem do abstrato para o concreto e das partes para o todo ${ }^{(11)}$. Debateu-se sobre as situações por elas vivenciadas com a pandemia, buscando 
ampliar seus conhecimentos, na perspectiva de admirar e explorar tais situações, suas percepções e dialogar sobre a CoVid-19, bem como sobre as preocupações geradas e planos modificados, conforme a Figura 2.

Figura 2 - Repercussões do enfrentamento da CoVid-19 na percepção das gestantes. As repercussões do enfrentamento da COVID-19 na vivência da gestação

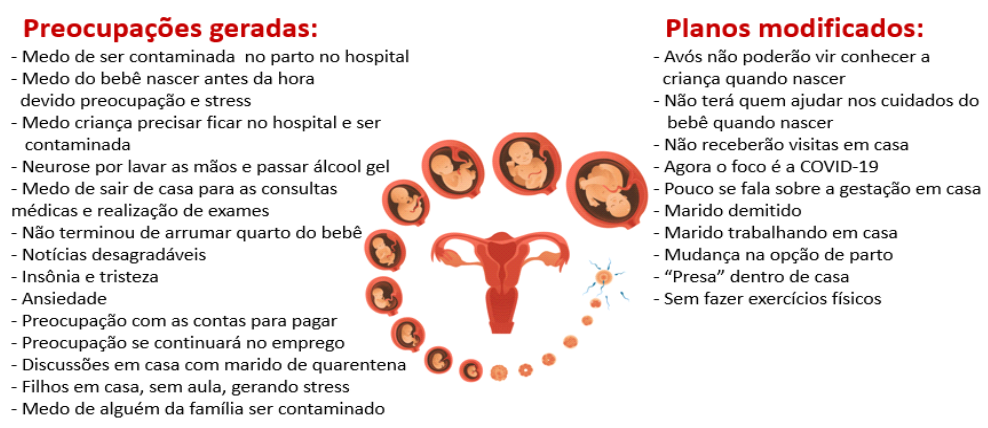

Fonte: Adaptado pelas autoras a partir de https://www.vectorstock.com/royalty-free-vector/human-embryo-development-circleflat-icon-vector-23329044(10)

Todas as preocupações apresentadas pelas gestantes foram discutidas no CCV, cabendo à mediadora desafiar cada vez mais as participantes, contextualizando a situação existencial codificada e as dúvidas que emergiram no diálogo. As gestantes foram participando e contribuindo com experiências e conhecimentos sobre os Temas Geradores e seus desdobramentos, promovendo a saúde umas das outras por meio do incentivo e do partilhar informações.

A última etapa do Itinerário foi 0 Desvelamento Crítico. Nessa etapa, também reconhecida como a fase da problematização, é desenvolvida a consciência por meio de conceitos. Os sujeitos envolvidos exteriorizam sua visão de mundo, e o mediador do Círculo procura reduzir sua intervenção direta no diálogo ao mínimo. Nesse momento se desenvolveu um processo de ação-reflexão sobre os Temas Geradores, que culminou com o revelar da sua consciência crítica ${ }^{(11)}$.

Aqui, o foco voltou-se para a busca da transformação das situações-problema vivenciadas pelas gestantes, com compreensão da realidade, por meio de reflexões sobre sua vida, desvelando os limites e as potencialidades. As gestantes tiveram a oportunidade de refletir sobre o aprendizado com a experiência do CCV e sobre o significado de vivenciar o enfrentamento da CoVid-19 no período gestacional. Elas apresentaram os significados, por meio de uma palavra-chave ou uma frase, avaliando sua vivência e aprendizado no Círculo, como demonstra a Figura 3.

Figura 3 - Aprendizado das gestantes na vivência do Círculo de Cultura Virtual.

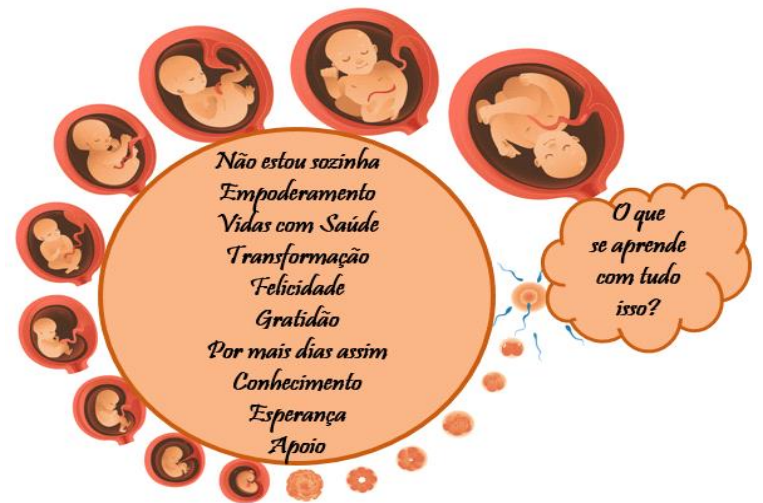

Fonte: Adaptado pelas autoras a partir de https://www.vectorstock.com/royalty-free-vector/human-embryo-development-circleflat-icon-vector-23329044(10)
Vale ressaltar
que as gestantes
durante o momento musical e convidaram demonstraram intensa emoção e apreciação, familiares para também participar: alguns 
cantaram junto, outros choraram, alguns se abraçaram e solicitaram que fosse entoada mais uma canção. A mediadora atendeu à solicitação e cantou a música 'Tocando em Frente', de Almir Sater, despertando profundas emoções nas gestantes e familiares (esposo e filhos).

No findar do CCV, as gestantes e alguns familiares (que se fizeram presentes no momento musical) verificaram a possibilidade de realizar outro encontro virtual. A mediadora, então, orientou que todas as gestantes e familiares descrevessem suas dúvidas e lhes encaminhar via mensagem WhatsApp, para essas temáticas serem discutidas no próximo Círculo. Todos os envolvidos no processo reagendaram outra data para interação, aprendizado, compartilhamento de informação e promoção da saúde mútua.

As reflexões no CCV foram norteadas pelo diálogo, necessário para torná-lo espaço dinâmico de aprendizagem para a discussão dos Temas Geradores e desvelamento do pensamento crítico-reflexivo das gestantes, o qual foi amplamente compartilhado.

Considerando ser a CoVid-19 uma situação nova no cenário mundial, com pesquisas ainda sendo realizadas e poucos estudos publicados a respeito, muitas orientações para seu enfrentamento são baseadas em doenças provocadas por outros vírus (SARS-CoV. MERSCoV; H1N1) pela semelhança e pela gravidade dos comprometimentos que determinam em gestantes, o que as classifica como grupo de risco $^{(12-13)}$.

Comumente, as alterações hormonais e emocionais da gravidez desencadeiam situações de estresse, medo e ansiedade. Na situação pandêmica atual, com a mulher gestante considerada grupo de risco, surgiram preocupações específicas como: o medo de sair de casa para as consultas de pré-natal, a possível contaminação de alguém de casa, a possibilidade de contaminar-se no hospital, de ter um parto prematuro, de o recém-nascido necessitar de hospitalização. Tudo isso se soma à ansiedade e ao estresse provocados pelo desconhecido, com as crianças em casa, com as discussões conjugais mais frequentes, o bombardeio de notícias desagradáveis sobre a CoVid-19, as preocupações financeiras e de estabilidade no trabalho, gerando também neuroses com o processo de higienização das mãos.

Cabe salientar que, durante a gestação, alguns fatores de risco podem desencadear depressão, tais como: história prévia de depressão, gravidez não planejada, ausência de parceiro ou de suporte social, condição elevada de estresse percebido, presença de adversidades, história de abuso ou violência doméstica, história passada ou presente de complicações obstétricas, perda fetal, problema financeiro, desemprego e dependência de substâncias psicoativas ${ }^{(14)}$.

As gestantes participantes dessa experiência relataram preocupações similares às apontadas como fatores de risco para a depressão. Deste modo, é imperativo o preparo da enfermagem e demais profissionais da área de saúde, para a prevenção da depressão durante a gestação, para planejamento de ações que ampliem a eficácia do cuidado prestado.

A pandemia de CoVid-19, assim como outras emergências de saúde pública, pode desencadear situações de altíssimo estresse, ansiedade e depressão em mulheres durante a gestação e gerar consequências negativas. Após o surto de CoVid-19, houve aumento na prevalência de depressão e, de modo geral, as gestantes ainda não diagnosticadas com depressão se tornaram mais suscetíveis ${ }^{(15)}$. Além disso, neste período de pandemia, as gestantes manifestam várias preocupações como não ir ao seu pré-natal pelo risco percebido de exposição, estresse, ansiedade e utilização constante e, por vezes, até excessiva de hipoclorito de sódio e álcool(16).

Na literatura há relato de perda de gravidez entre o segundo e o terceiro trimestre de gestação de mulheres afetadas por CoVid-19, com condição clínica grave e internação em unidade de terapia intensiva. No entanto, a preocupação é maior com o trabalho de parto prematuro, que atinge mais as mulheres afetadas pela CoVid-19 ${ }^{(17-19)}$, bem como com o sofrimento fetal(20). Esse fato condiz com a preocupação de contrair a doença e de parto prematuro manifestada pelas participantes do CCV.

Associado ao medo de serem contaminadas no hospital, as gestantes participantes deste estudo manifestaram preocupação com a possibilidade de a criança ficar hospitalizada ao nascer, seja pela prematuridade ou pela possível contaminação com CoVid-19. No entanto, os desfechos neonatais evidenciam manifestações clínicas relacionadas com a prematuridade, tendo em vista que a maioria dos recém-nascidos testados para a CoVid-19 tiveram resultados negativos. Os que nasceram com mais de 36 semanas apresentavam-se bem na alta hospitalar, enquanto alguns prematuros, com mais de 31 semanas, necessitaram de internação em unidade 
de terapia intensiva neonatal e a morbidade está relacionada com a maior prematuridade. Assim, nos estudos descritos até o momento, não é possível inferir que há transmissão vertical da CoVid-19 $9^{(17,19)}$.

Diante de tantas inquietações e desconhecimentos sobre os efeitos da CoVid-19, na gestação e no distanciamento social imposto às mulheres, o emprego de recursos disponíveis, como a música nesta experiência, pode contribuir para promover a saúde, pois, além de ser uma linguagem universal, a música possibilita momentos de diversão, redução do stress, facilita o vínculo, oportuniza reflexão pelas mensagens emitidas, desperta emoções, lembranças, bom humor, entre outros benefícios ${ }^{(21)}$.

É também importante argumentar sobre a disponibilização de espaços para o cuidado online, que se torna uma ferramenta de apoio às mulheres durante este período, oferecendo acolhimento e espaço para trocas com outras gestantes que vivenciam as mesmas situações, uma vez que estão privadas de atividades em grupo, habitualmente disponibilizadas pela rede de atenção à saúde. Tais práticas possibilitam o empoderamento das gestantes, auxiliando-as a identificar suas próprias necessidades, controlar o estresse, a fadiga e a enfrentar de modo mais eficaz suas preocupações e mudanças de planos em tempos de estresse ${ }^{(22)}$. Esta ferramenta é importante para reduzir níveis de estresse, que podem promover a saúde geral e a qualidade de vida ${ }^{(23)}$.

Nesta situação de isolamento social, é necessário criar canais de comunicação para gestantes e disponibilizar suporte on-line em tempo real para fornecer apoio emocional e orientações eficazes. É importante considerar a implementação de assistentes virtuais que consigam responder às dúvidas frequentes e proporcionar a participação de mulheres que vivenciam a mesma situação. As universidades desempenham papel fundamental e devem envolver professores e estudantes, de modo articulado e coordenado, para fornecer esse suporte por meio da web ${ }^{(24)}$.

Desta forma, o CCV, fundamentado no Itinerário metodológico de Paulo Freire, constituise um processo educativo e participativo, abrangendo o contexto social, com estabelecimento de vínculos entre gestantes e enfermeiras, com a troca construtiva capaz de modificar o meio por ações e orientações, construindo um processo problematizador baseado na realidade de cada participante. $\mathrm{Na}$ metodologia de Freire, o indivíduo torna-se ativo e participante por meio do diálogo e dos debates em grupo, tornando-se crítico e reflexivo diante de situações reais que o desafiam, possibilitando intervir neste contexto em que todos os participantes participam lendo e escrevendo o mundo com suas próprias idéias ${ }^{(25)}$.

Diante das preocupações geradas e dos planos modificados pela pandemia da CoVid-19, constatou-se o aprendizado das gestantes na vivência do CCV. A proposta de educação libertadora proposta por Freire pode ser entendida como forma de compreender o mundo, refletir sobre ele, transformando a realidade a partir de uma ação consciente. Seu pensamento tem colaborado de forma significativa na construção de conhecimentos, a partir de uma atitude crítica e reflexiva na Enfermagem, incorporando a educação problematizadora, tendo como ponto de partida o diálogo com os educandos ${ }^{(25)}$.

Diante da experiência exitosa no CCV, que oportunizou a integração e o contato social a distância com mulheres que vivenciam a mesma situação e desenvolvem sentimentos semelhantes, vislumbra-se que o espaço virtual pode configurar-se como uma tecnologia educativa para a Enfermagem, em prol da promoção da saúde na comunidade. Além disso, possibilita acolhimento, troca de experiências e de informações, diálogo horizontal, construção do saber e liberdade de expressão, mesmo as participantes estando distantes, geograficamente.

Esta experiência teve como limitação o fato de ter sido realizada somente com as gestantes, com participação do companheiro e de outros membros da família apenas ao término, no processo de reflexão. Além disso, o desenvolvimento do Círculo de Cultura em espaço virtual foi uma proposta inovadora, merecendo novos olhares e aprimoramento da estratégia, principalmente diante da necessidade de restrição social como medida de prevenção da CoVid-19. Esses ensaios demonstram a relevância de investigar as percepções de outros públicos quanto ao enfrentado nesta crise, no Brasil e mundo.

\section{CONCLUSÃO}

A vivência de um CCV, com reflexões sobre o enfrentamento da Coronavirus Disease 2019, ressignificou a percepção de gestantes sobre as 
repercussões da doença para a sua saúde e a dos seus bebês.

Cabe sinalizar que a limitação de acesso a tecnologias de comunicação, como o computador e a própria rede de internet, constitui fator dificultador para replicação desta experiência com alguns grupos de gestantes.

A educação do século XXI vem passando por mudanças e inovações constantes, que promovem o estabelecimento de relações da teoria e da prática, por intermédio de diferentes métodos e tecnologias. O CCV contribuiu, não somente como um dispositivo de educação que pode ser utilizado por profissionais da saúde, em diferentes oportunidades, mas também na transformação das práticas de cuidado, valorizando a experiência das participantes e promovendo seu empoderamento.

\section{REFERÊNCIAS}

1- Gernand AD, Schulze KJ, Stewart CP, West KP, Christian P. Micronutrient deficiencies in pregnancy worldwide: Health effects and prevention. Nat Rev Endocrinol. 2016;12(5):27489. DOI: $10.1038 /$ nrendo.2016.37

2- World Health Organization (WHO). Recomendações sobre cuidados pré-natais para uma experiência positiva na gravidez. Geneva: World Health Organization; 2016 [cited 2020 July 20]. Available in: https://www.who.int/reproductivehealth/publica tions/anc-positive-pregnancy-experiencesummary/pt/

3- Wang D, Hu B, Hu C, Zhu F, Liu X, Zhang J, et al. Clinical characteristics of 138 hospitalized patients with 2019 Novel Coronavirus-Infected Pneumonia in Wuhan, China. JAMA 2020;323(11):1061-9. DOI: 10.1001/jama.2020.1585

4- Zhu N, Zhang D, Wang W, Li X, Yang B, Song J, et al. A novel coronavirus from patients with pneumonia in China, 2019. N Engl J Med. 2020;382(8):727-33.

DOI:

\subsection{6/NEJMoa2001017}

5- Brasil. Ministério da Saúde. Protocolo de manejo clínico da CoVid-19 na Atenção Especializada. Brasília: Ministério da Saúde; 2020. 6- Dickinson $F$, McCauley $M$, Smith $H$, van den Broek N. Patient reported outcome measures for use in pregnancy and childbirth: A systematic review. BMC Pregnancy Childbirth 2019;19:155. DOI: 10.1186/s12884-019-2318-3

7- Heidemann ITSB, Dalmolin IS, Rumor PCF, Cypriano CC, Costa MFBNA, Durand MK. Reflexões sobre o itinerário de pesquisa de Paulo Freire:
Contribuições para a saúde. Texto ContextoEnferm. 2017;26(4):1-8. DOI: 10.1590/010407072017000680017

8- Silva KJ, Vendruscolo C, Silva Filho CC, Durand MK. Círculo de cultura como espaço dialógico na enfermagem: Um método transformador. In: Bággio $V$, organizador. Vozes da educação: Uma partilha de educadores e educadoras que refletem sobre suas vivências cotidianas em diferentes espaços pedagógicos. 2a ed. Veranópolis: Diálogo Freireano; 2019. v. 2. p. 237-50

9- Dalmolin IS, Heidemann ITSB, Freitag VL. Práticas integrativas e complementares no Sistema Único de Saúde: Desvelando potências e limites. Rev Esc Enferm USP 2019;53:1-8. DOI: 10.1590/s1980-220x2018026603506

10- Human embryo development circle flat icon vector image. Available in:

https://www.vectorstock.com/royalty-freevector/human-embryo-development-circle-flaticon-vector-23329044

11- Freire P. Pedagogia do oprimido. 64a ed. Rio de Janeiro: Paz e Terra; 2015.

12- Alserehi H, Wali G, Alshukairi A, Alraddadi B. Impact of Middle East Respiratory Syndrome coronavirus (MERS-CoV) on pregnancy and perinatal outcome. BMC Infect Dis. 2016;16:105. DOI: 10.1186/s12879-016-1437-y

13- Assiri A, Abedi GR, Al Masri M, Bin Saeed A, Gerber SI, Watson JT. Middle East Respiratory Syndrome Coronavirus Infection during pregnancy: A report of 5 cases from Saudi Arabia. Clin Infect Dis. 2016;63(7):951-3. DOI: 10.1093/cid/ciw412

14- Biaggi A, Conroy S, Pawlby S, Pariante CM. Identifying the women at risk of antenatal anxiety and depression: A systematic review. J Affect Disord. 2016;191:62-77. DOI: 10.1016/i.jad.2015.11.014

15- Wu Y-T, Zhang C, Liu H, Duan C-C, Li C, Fan J-X, et al. Perinatal depression of women Along with 2019 novel Coronavirus breakout in China. SSRN Electr J. 2020:1-30. DOI: 10.2139/ssrn.3539359

16- Fakari FR, Simbar M. Coronavirus pandemic and worries during pregnancy: A letter to editor. Arch Acad Emerg Med. 2020 [cited 2020 Apr 14]; 8(1):e21. Available in: https://www.ncbi.nlm.nih.gov/pmc/articles/PMC $\underline{7075675 /}$

17- Chen H, Guo J, Wang C, Luo F, Yu X, Zhang W, et al. Clinical characteristics and intrauterine vertical transmission potential of COVID-19 infection in nine pregnant women: A retrospective review of medical records. Lancet 
2020;395(10226):809-15. DOI: $10.1016 /$ So1406736(20)30360-3

18- Liu Y, Chen H, Tang K, Guo Y. Clinical manifestations and outcome of SARS-CoV-2 infection during pregnancy. J Infect. 2020. DOI: 10.1016/j.jinf.2020.02.028

19- Zhu H, Wang L, Fang C, Peng S, Zhang L, Chang $G$, et al. Clinical analysis of 10 neonates born to mothers with 2019-nCoV pneumonia. Transl Pediatr. 2020;9(1):51-60. DOI: $\underline{10.21037 / \mathrm{tp} .2020 .02 .06}$

20- Wang $X$, Zhou Z, Jianping Z, Zhu F, Tang Y, Shen $X$. A case of 2019 Novel Coronavirus in a pregnant woman with preterm delivery. Clin Infect Dis. 2020. DOI: $10.1093 /$ cid/ciaa200

21- Souza JB, Martins EL, Xirello T, Urio $A$, Barbosa SSP, Pitilin EB. Interface entre a música e a promoção da saúde da mulher. Rev Bras Promoç Saúde 2020;33:1-10. DOI: 10.5020/18061230.2019.9466

22- Chau V, Giallo R. The relationship between parental fatigue, parenting self-efficacy and behaviour: Implications for supporting parents in the early parenting period. Child Care Health Dev. 2015;41(4):626-33. DOI: $10.1111 / c c h .12205$

23- Holtz B, Smock A, Reyes-Gastelum D. Connected motherhood: Social support for moms and moms-to-be on Facebook. Telemed J E Health 2015;21(5):415-21. DOI: 10.1089/tmj.2014.0118

24- Bermejo-Sánchez FR, Peña-Ayudante WR, Espinoza-Portilla E. Depresión perinatal, COVID-19 y redes sociales. Acta Méd Peru 2020;37(1):88-93. DOI: $10.35663 / a m p .2020 .371 .913$

25- Freire P. Educação como prática de liberdade. São Paulo: Paz e Terra; 2019.

Nota: O estudo foi desenvolvido sem financiamento.

Recebido em: 20/05/2020

Aprovado em: 09/09/2020

Endereço de correspondência:

Jeane Barros de Souza

Universidade Federal da Fronteira Sul - UFFS. Rodovia SC 484

- Km 02. Bairro Fronteira Sul. Chapecó SC. CEP: 89.815-899.

E-mail: jeanebarros18@gmail.com 